\title{
Città diverse
}

\section{Gabriele Paolinelli}

Dipartimento di Architettura, Università degli Studi di Firenze, Italia gabriele.paolinelli@unifi.it 
L'importanza della diversità biologica vegetale e animale della vita selvatica è argomentata da una letteratura ampia e stratificata. Anche del domestico si considera la diversità vegetale e animale di cui gli uomini fanno uso da migliaia di anni. In entrambi i casi la perdita di specie e varietà è il principale fattore di sollecitazione scientifica, tecnica e politica. È logico che non si conosca tutto il vivente ed è biologico che compaiano e scompaiano specie. La biodiversità è connessa sia all'evoluzione, che all'estinzione, nell'avvicendarsi spazio-temporale della vita. La conoscenza scientifica e la consapevolezza culturale di ciò consente di avere un appropriato rispetto di dinamiche che comprendono la presenza umana sulla Terra in processi superiori, rispetto ai quali è una delle variabili complesse che interagiscono. Ciò non cambia però il significato e la rilevanza di due fatti attestati dalle conoscenze. II primo è appunto relativo alla perdita di diversità biologica: progredisce, nonostante le percezioni e le azioni umane che sempre più vengono volte al suo contrasto. Più che un tema d'inefficacia, per cui tali scelte potrebbero essere considerate in sé inutili, c'è una questione di improprio bilanciamento tra quanto viene fatto a scapito della conservazione della biodiversità, rispetto a ciò che è a suo favore. $\mathrm{E}$ questo porta al secondo fatto: le attività umane condizionano la vita selvatica a causa della frammentazione, della riduzione e dell'eliminazione di habitat.
Le pratiche industriali di coltivazione e allevamento hanno inoltre generato una marcata semplificazione della ricchezza specifica della vita vegetale e animale domestica.

Questa narrazione ha una decisa similitudine con quella del cambiamento climatico, che è intrinseco alla storia del pianeta, ma ha componenti causali antropiche non trascurabili. Anche per la perdita di specie di organismi viventi, è pertanto chiaro che nascondere dietro alla indeterminatezza e alla mutevolezza naturale le inadeguatezze culturali di gran parte delle società umane è ideologico, difetta di senso etico e si pone fuori da ogni concreta prospettiva di sostenibilità.

Se si riferisce quanto fin qui considerato alla pertinenza del progetto emerge un'indicazione. Progettare ha a che fare con il volgere il pensiero e l'immaginazione, le tecniche e le tecnologie, verso la generazione di condizioni di vita favorevoli, con proprietà adattative, combinando conservazione e trasformazione delle realtà date. Di biodiversità occorre pertanto occuparsi con il progetto, tanto per limitare e mitigare le azioni umane che ne comportano la riduzione, quanto per promuovere e diffondere quelle che possono sostenerne la conservazione e l'incremento, caso per caso, luogo per luogo, comunità per comunità. II progetto è infatti vincolato alle scale del luogo e del paesaggio; agisce di- 


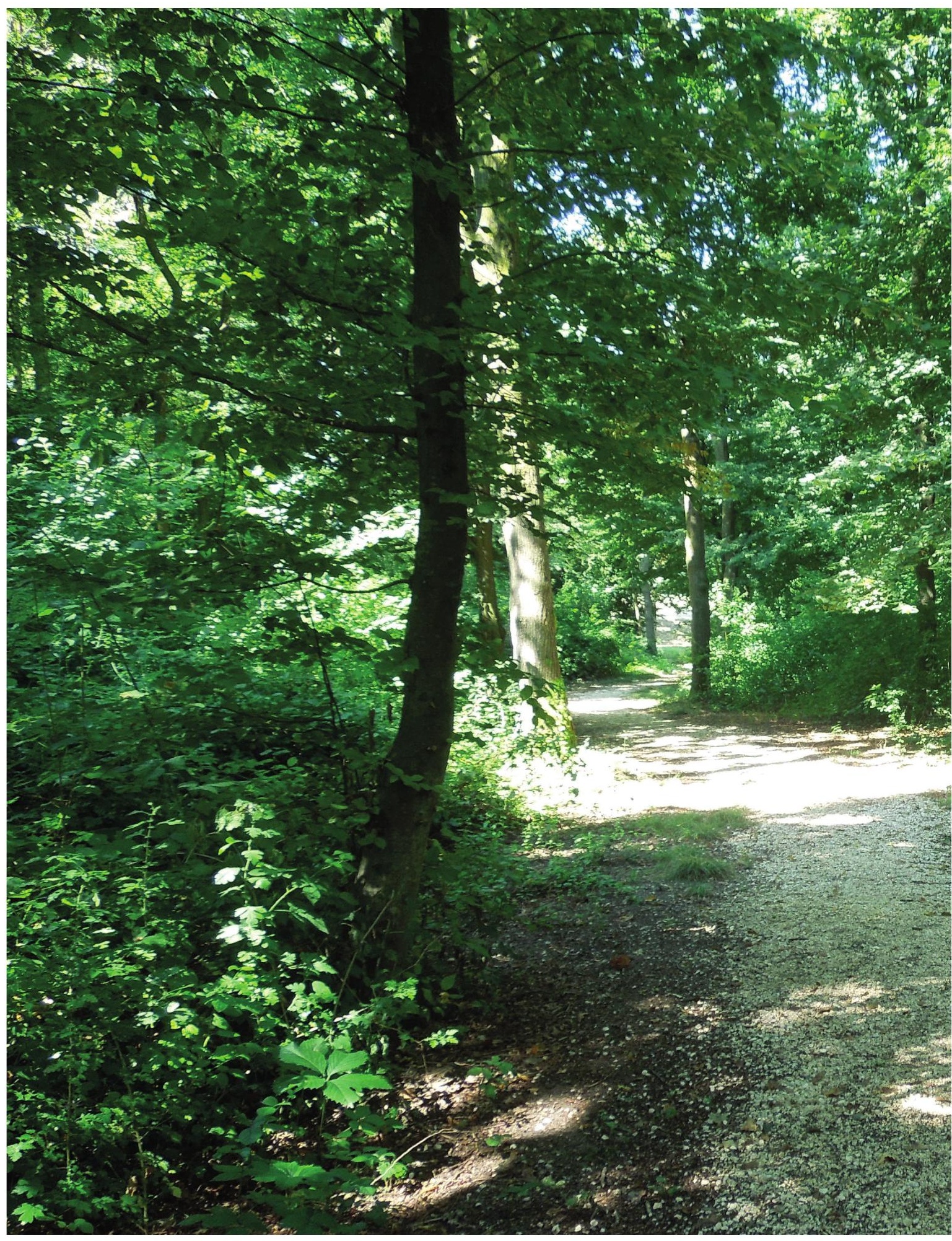

Fig. 1 - Bosco dell'Osellino, Mestre - foto di Emma Salizzoni 

(4)

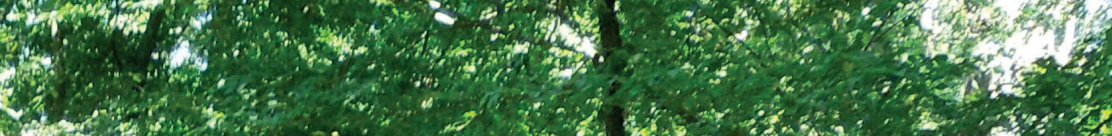

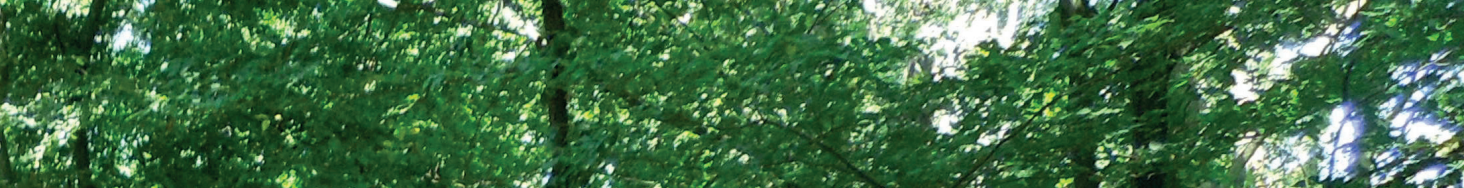

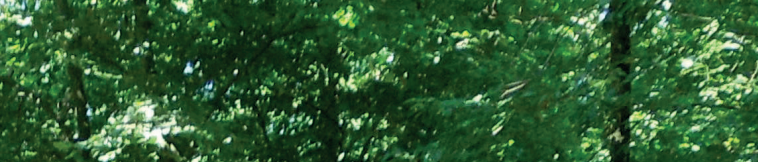

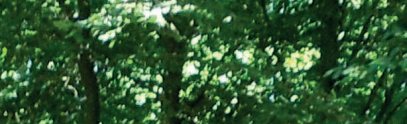
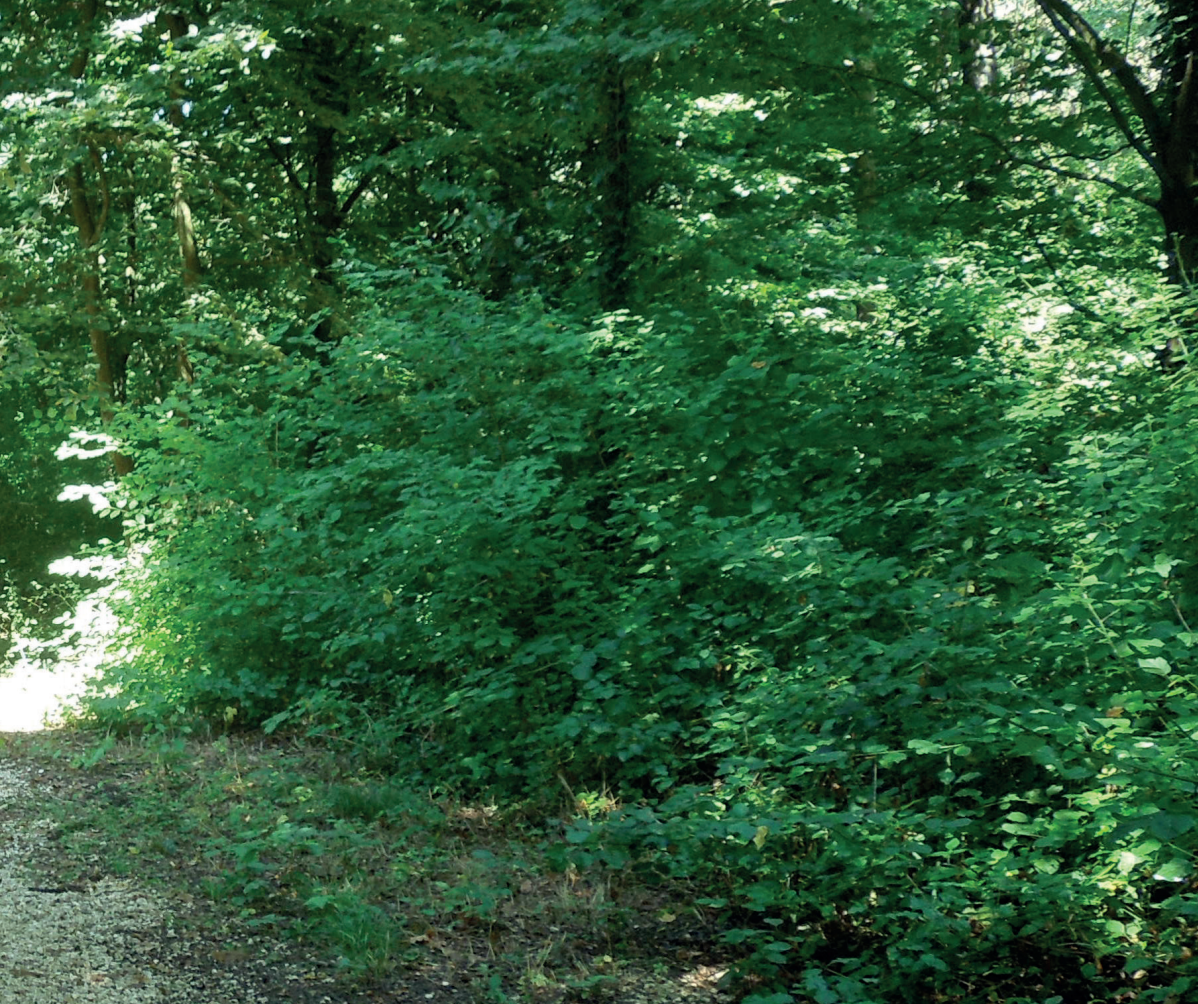

galopoli' con flussi di materia, energia e informazione sempre più intrecciati in estese relazioni di interdipendenza. La biodiversità delle città non è pertanto l'ennesimo vezzo che l'urbano volge al rurale o l'artificiale al naturale. Ciò ha piuttosto a che fare con una reciprocità di bisogni e benefici possibili, della città nei confronti della biodiversità e di questa nei confronti della città. Quelle di cui si discute sono città diverse da quanto si è finora immaginato, costruito, trasformato. Dagli ultimi due decenni del secolo scorso e in particolare durante i primi tre di quello corrente, che oggi pensa il 2030 al tempo presente, si stanno sviluppando concezioni ed azioni diverse da quelle che hanno connotato le trasformazioni urbane per più millenni. Si tratta di spostamenti contenuti, ma significativi per le divergenze che esprimono.

$\dot{E}$ in questo contesto che con Nature in città. Biodiversità e progetto di paesaggio in Italia vengono proposte risorse informative e visioni critiche, e dunque anche risorse formative. II libro offre più chiavi interpretative composte in uno strumento agile e al tempo stesso nutrito da un ampio corredo bibliografico; questa riflessione ne propone alcune emerse da una lettura, ma altre potranno essere trovate e messe a fuoco nei loro significati.

"Coabitazioni" è il termine principale attraverso cui ed ai significati particolari dei contributi che la articolano. Diversa dalla coesistenza, più o meno tollerante, la coabitazione è relativa al condividere gli habitat in relazioni di prossimità e reciprocità con altri esseri viventi, nel contesto specifico in cui il libro si colloca, con organismi di altre specie. II termine, inoltre, contiene al tempo stesso una consapevolezza ed una prospettiva: la biodiversità non è estranea alla città e la città può anche essere pensata a favore della biodiversità. Ciò non stupirà chi ha affrontato palesi e resistenti idiosincrasie rispetto alla visione della città come paesaggio, rispetto alla quale è intrinseco quanto adesso appare innovativo. La storia della biodiversità urbana non è in fondo diversa da quella dei suoli urbani, dai quali peraltro dipende.

Risulta così preziosa la disamina della questione attraverso più ottiche della pianificazione spaziale. Oltre al pregevole contributo informativo recato dal volume, più rilievi critici rivestono in esso un'importanza evidente mano a mano che con la lettura si dipana un'intricata realtà di strumenti, norme, procedimenti, competenze, approcci, dati. Da tanto impegno di ordinamento emerge di fatto un generalizzato stato confusionale, nel quale vengono però identificate possibili direzioni di evoluzione chiare, potenzialmente penetranti. Il tema della "transizione da quantità a qualità" costituisce in tal senso un'altra chiave con la quale è possibile spostare l'attenzione verso una visione di città diversa, intelligente e lungimirante, che mette a nudo la categoria del "verde urbano", rivelandone la sua reale natura di “urbanizzazione". Da una categoria ad un'altra, la "foresta urbana" riceve altrettanta attenzione, nei citati termini dell'insufficienza di una visione per sole quantità, ma anche rispetto a quelli della parzialità di un bilancio per soli servizi, laddove insieme ai benefici non siano considerati gli svantaggi. In generale le teorie e le applicazioni di forestazione urbana non indulgono nel compiacimento semplicistico per un generalizzato "inselvatichimento" della città. 


\section{NATURE IN CITTÀ}

Biodiversità e progetto di paesaggio in Italia

a cura di

Alessandro Gabbianelli

Bianca Maria Rinaldi

Emma Salizzoni

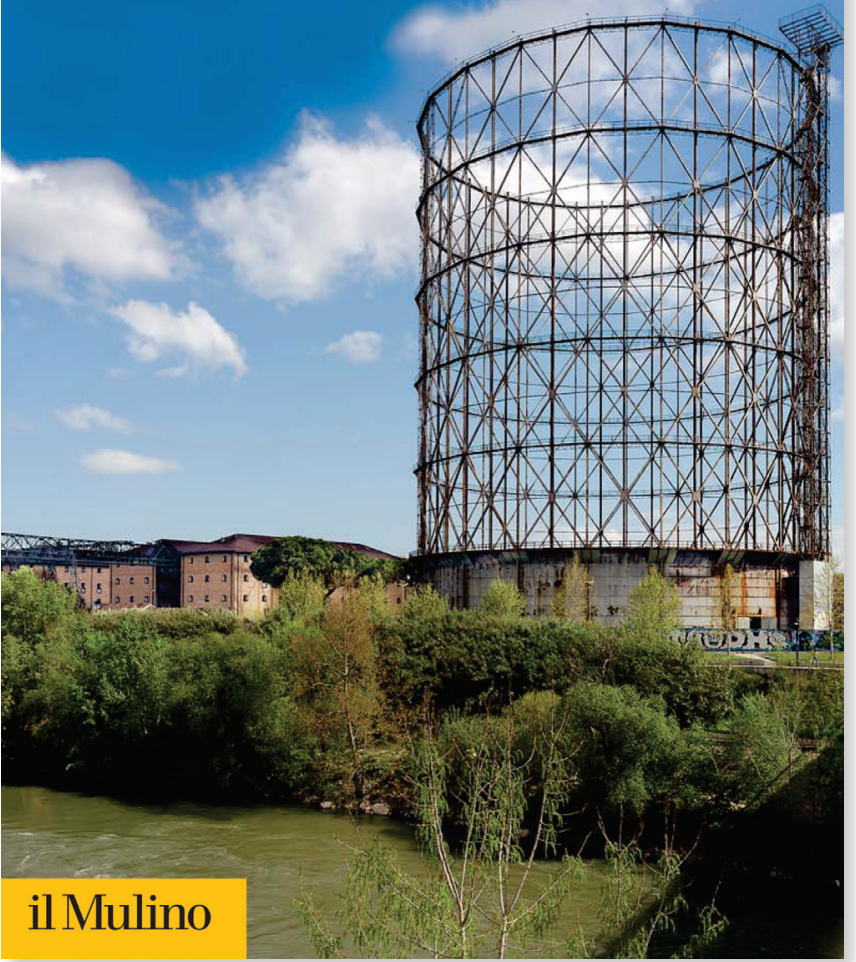

Nature in città. Biodiversità e progetto di paesaggio in Italia,

il Mulino, Bologna, 2021.

A cura di:

Alessandro Gabbianelli, Bianca Maria Rinaldi, Emma Salizzoni.

Con contributi di:

Claudia Canedoli, Claudia Cassatella, Alessandro Gabbianelli, Paola Cullino, Federica Larcher, Emilio Padoa-Schioppa, Bianca

Maria Rinaldi, Manuela Ronci, Noemi Rota, Emma Salizzoni. 


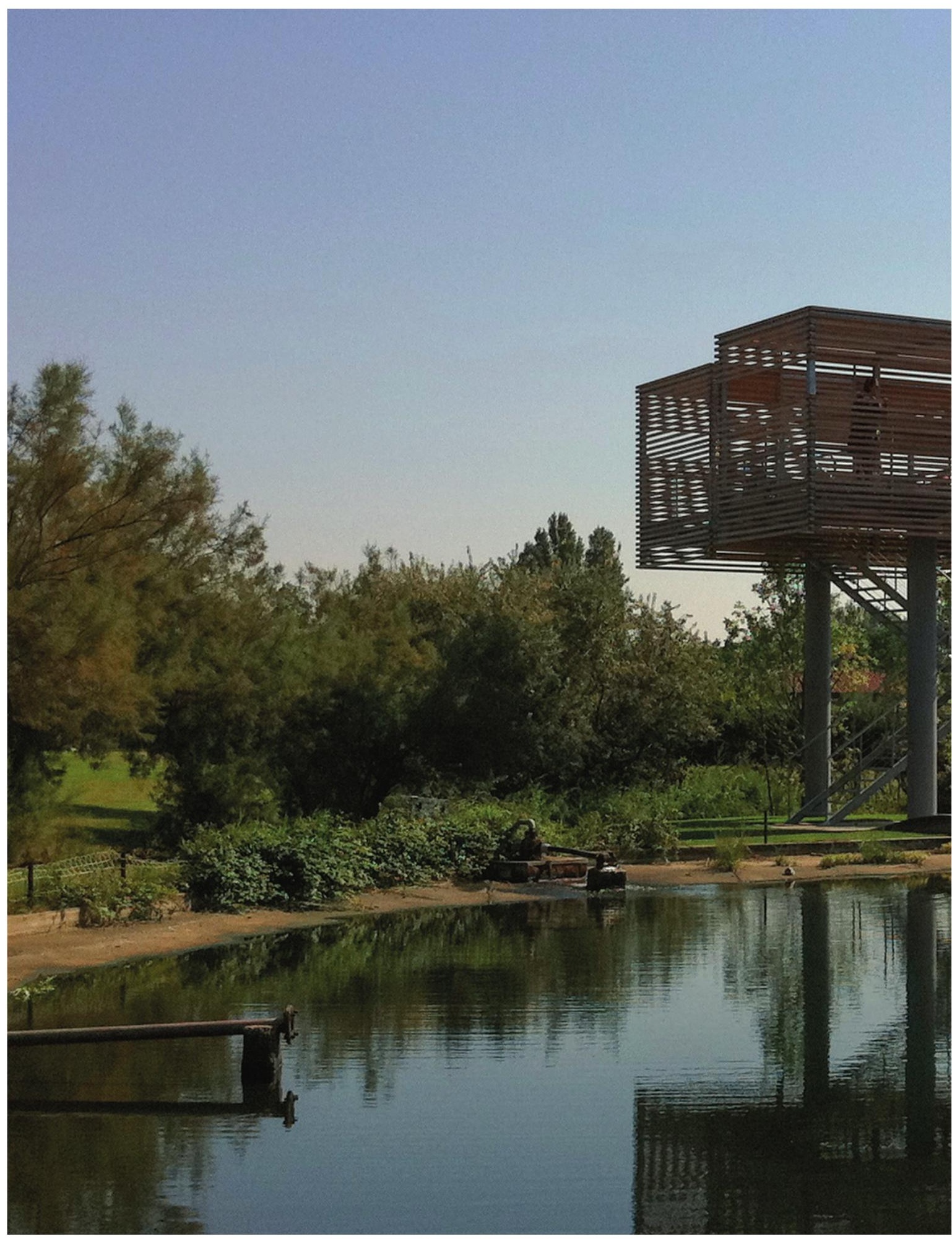

Fig.2 - Isola di Arbarella, Rovigo (I'Airone, Anna Braioni) - foto di Anna Braioni 
Poiché però il tema è molto esposto anche a livello culturale e politico, ed è opportuno che lo sia il più possibile, ha un'importante valenza divulgativa il richiamo a porre attenzione critica alla questione. Attraverso un'adeguata diversità scientifica, capace di interagire nei processi, è possibile sviluppare e gestire una significativa diversità biologica nei paesaggi, tanto più in quelli delle città. Ciò potrà ulteriormente contribuire a sviluppare le dimensioni "interdisciplinare" e "transdisciplinare" che la progettazione paesaggistica ha radicato nelle proprie teorie e prassi. Si è pertanto di fronte ad un'opportunità reciproca, di utilità della disciplina ai fini della conservazione e dello sviluppo della biodiversità, quanto di quest'ultima come stimolo per l'arricchimento ed il rafforzamento scientifico e tecnico, teorico e pratico. Una pur accorta considerazione della contemporaneità può concludere ancora poco su cosa è e potrà essere la "progettazione ecologica urbana", mentre sappiamo cos'è l'architettura del paesaggio: una disciplina che comprende nella progettazione la concezione ecologica degli interventi. Quel che è evidente e certamente vale anche per la progettazione ecologica urbana è che c'è bisogno di approcci sintetici, che portino a significato operativo le molteplici visioni specialistiche necessarie, senza che esse divengano responsabili di azioni settoriali. Cosa ha a che fare poi I'“estetica" - ecco un'altra chi- le specie degli organismi viventi che connota un certo paesaggio? La risposta è proprio in questo ultimo termine, che con il primo ha ampie e variamente discusse relazioni essenziali. Le caratteristiche di biodiversità sono anche in buona parte percepibili dagli esseri umani ed in quanto tali interpretate in termini estetici, influenzando tanto la considerazione di una determinata parte di città, quanto l'evoluzione dello stesso senso estetico della cultura di un determinato contesto di spazio e tempo.

Questa proiezione verso città biologicamente diverse, esteticamente percepite, trova suggestioni anche nelle connotazioni biologiche dei siti archeologici, dove la diversità della vita si fa "monumentale". II delicato rapporto tra le sue espressioni biologiche e la conservazione fisica della preziosa consistenza testimoniale dei beni archeologici fornisce inoltre un esempio del tema della coabitazione, peculiare, ma al tempo stesso indicativo anche per i molti casi in cui esso si pone invece in luoghi che appartengono al quotidiano.

Luoghi comuni, questi ultimi, dove la coabitazione si basa sul rispetto delle esigenze specifiche degli abitanti. La concezione progettuale della "distanza" che lo consente diviene così essenziale per unire, più che per separare, per consentire la condivisione dello stesso habitat nell'intento di massimizzare i benefici reciproci, contenendo meglio possibile i disturbi. 
La lettura ha portato alla memoria dello ha-ha romantico, efficace soluzione spaziale per delimitare il parco nel paesaggio attraverso un'interposizione che non ostacola la reciproca continuità percettiva. La questione è diversa, ma l'approccio spaziale di distanziamento suggerisce una qualche similitudine. Sono comunque distanze diverse interposte dal progetto quelle che consentono tanto l'"osservazione", quanto l'"esplorazione"; attraverso i filtri della percezione, esse non danno accesso al solo godimento estetico, ma anche alla conoscenza dei luoghi e alla connessa comprensione di alcune cose della vita naturale e della sua diversità. II progetto colloca e definisce "dispositivi spaziali" che favoriscono l'osservazione e l'esplorazione, ma anche le condizionano, esercitando dunque un controllo preventivo dei comportamenti umani rispetto a successioni ecologiche secondarie che hanno comunque vulnerabilità.

Queste realtà sono assunte come "ordinarie" nell'evoluzione contemporanea dei paesaggi urbani, parti del quotidiano, divengono "familiari" attraverso le relazioni che il progetto genera. L'ordinario può risultare perfino "iconico", quando la sua esperienza trasfigura uno o più elementi comuni in qualcosa capace di esprimere una carica estetica imprevista, mentre "salvifico" laddove il progetto e l'opera trasmettono messaggi culturali con espliciti accenti sui benefici che i luoghi forniscono.
II libro si chiude con un'altra chiave essenziale in architettura del paesaggio, quella della "partecipazione", che molto ha a che fare con la coabitazione con cui è stato aperto. La partecipazione facilita infatti la comprensione del diverso, del selvatico in questo caso, e consente in tal modo di capire come è possibile abitare la città insieme agli altri esseri viventi che ne compongono la diversità biologica.

Con la lettura di questo libro, il cui profilo scientifico non è mai appesantito dai numerosi quanto selezionati riferimenti alla letteratura, si studiano nuove cellule di nuovi tessuti della città contemporanea. La concezione che ciò può promuovere vede gli esseri umani come beneficiari in comodato d'uso, piuttosto che proprietari, condomini, piuttosto che individui e nuclei sociali isolati in recinti illusoriamente protetti. Questo è un libro da leggere, su cui tornare, per consultarne le parti e interrogarle, per comporre risposte a questioni specifiche o semplicemente per vivere in modo diverso, in città diverse. Dunque è anche un libro che può facilmente trascendere il campo scientifico da cui nasce, favorendo ulteriori contaminazioni utili; ma ciò che è altrettanto auspicabile, contando sulla scorrevolezza della scrittura che offre, è che divenga uno strumento di largo uso per lo sviluppo di un esteso pensiero critico trasversale sulla contemporaneità, sempre più evidentemente necessario per la sua sostenibilità. 


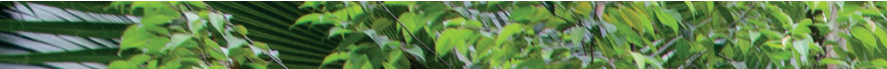

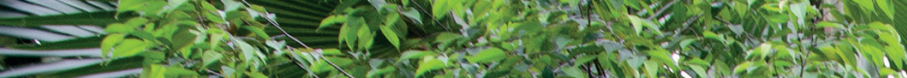

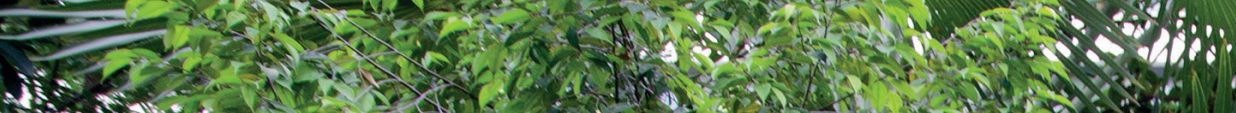

S.

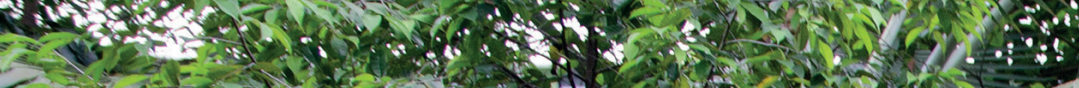

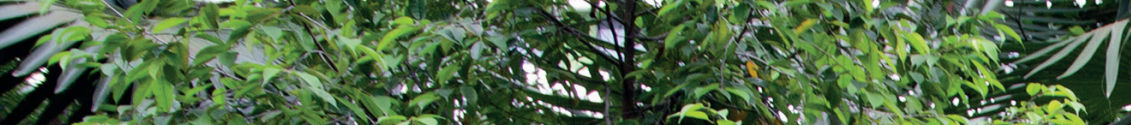

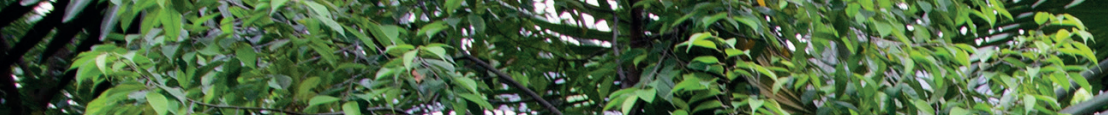

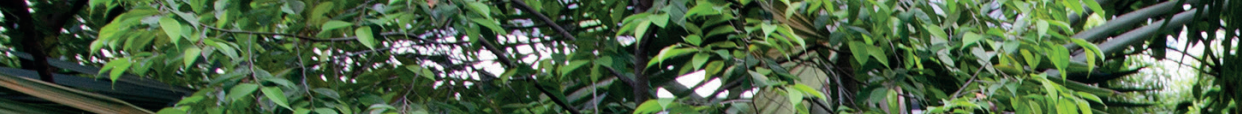
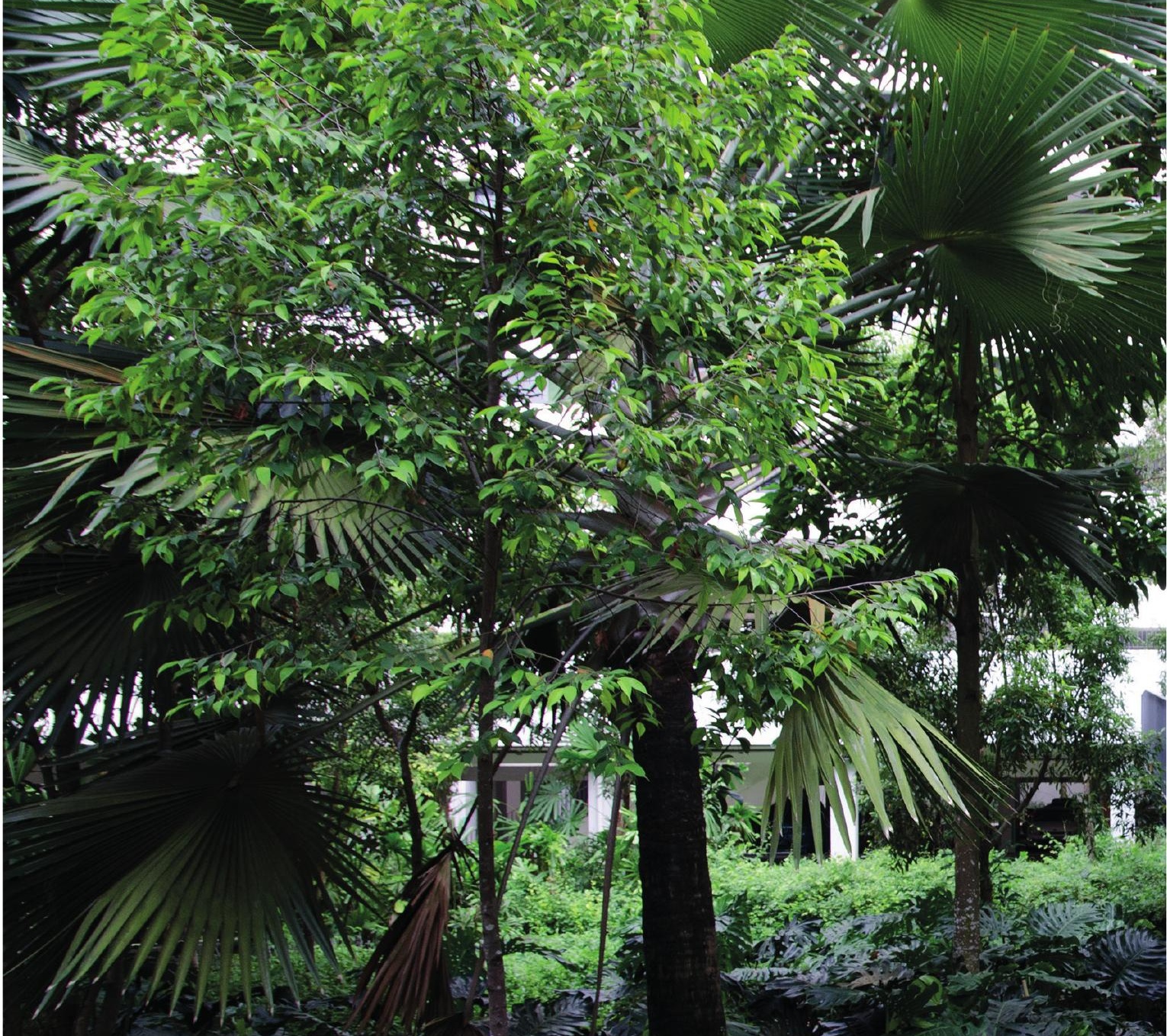

(n)

1.

4200

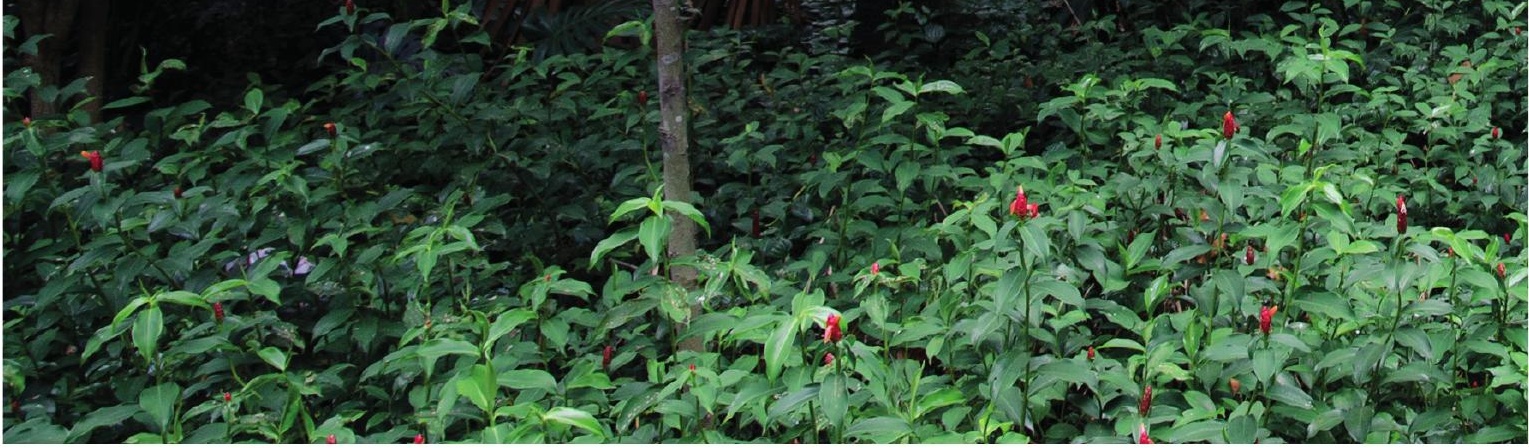

Fig.3 - Punggol New Town, Singapore (Punggol Waterway Terraces, ICN Design) - foto di Bianca Maria Rinaldi 


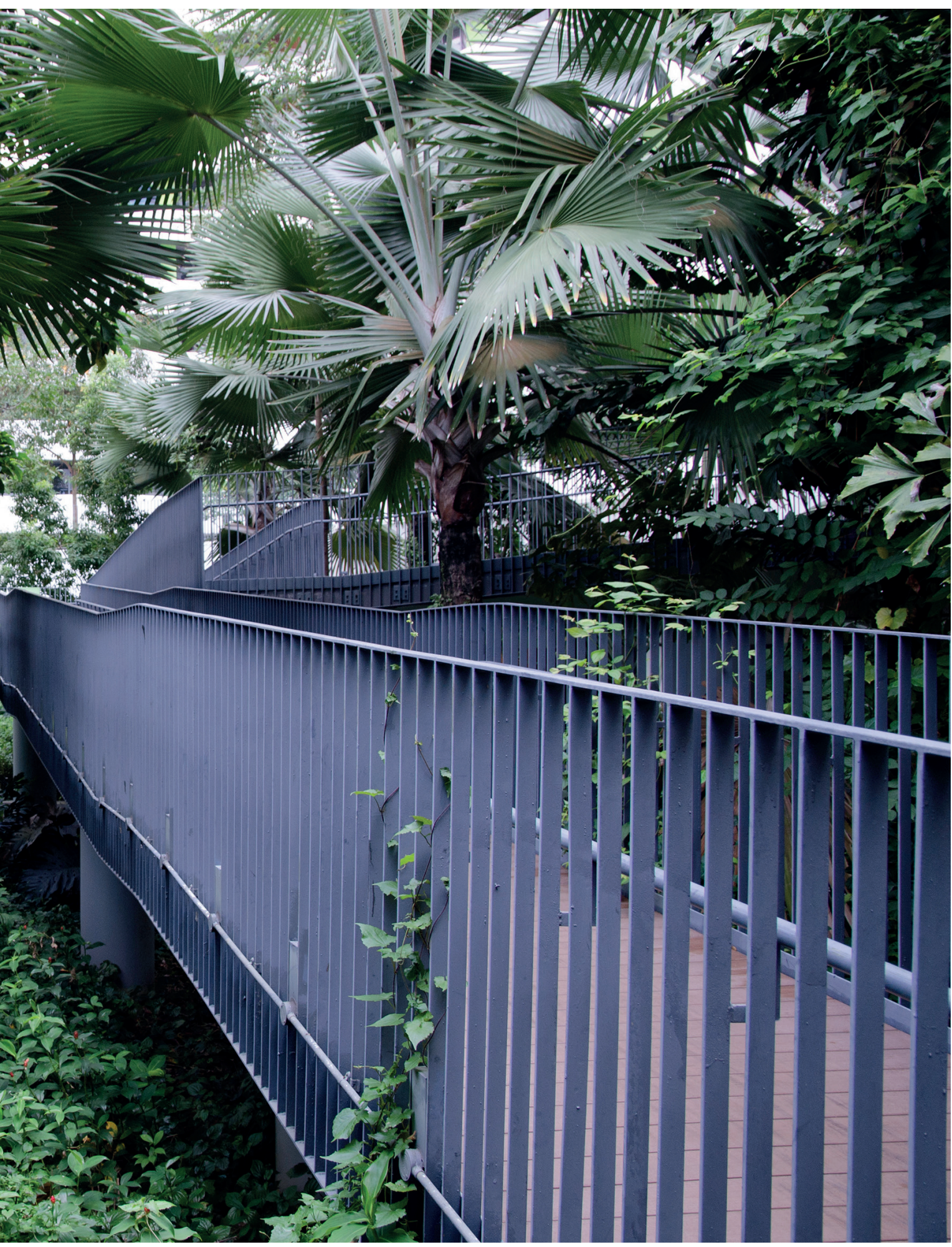

